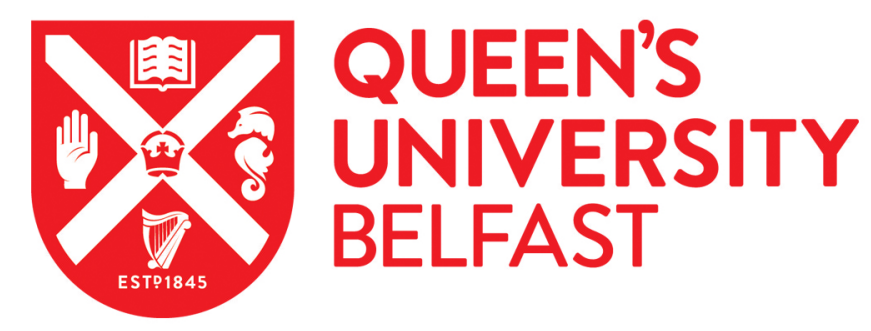

\title{
Multi-wavelength observations of the 2014 June 11 M3.9 flare: temporal and spatial characteristics
}

Christian, D. J., Kuridze, D., Jess, D. B., Yousefi, M., \& Mathioudakis, M. (2019). Multi-wavelength observations of the 2014 June $11 \mathrm{M} 3.9$ flare: temporal and spatial characteristics. Research in Astronomy and Astrophysics, 19(7), [101]. https://doi.org/10.1088/1674-4527/19/7/101

Published in:

Research in Astronomy and Astrophysics

Document Version:

Peer reviewed version

Queen's University Belfast - Research Portal:

Link to publication record in Queen's University Belfast Research Portal

Publisher rights

Copyright 2019 IOP. This work is made available online in accordance with the publisher's policies. Please refer to any applicable terms of use of the publisher.

\section{General rights}

Copyright for the publications made accessible via the Queen's University Belfast Research Portal is retained by the author(s) and / or other copyright owners and it is a condition of accessing these publications that users recognise and abide by the legal requirements associated with these rights.

Take down policy

The Research Portal is Queen's institutional repository that provides access to Queen's research output. Every effort has been made to ensure that content in the Research Portal does not infringe any person's rights, or applicable UK laws. If you discover content in the Research Portal that you believe breaches copyright or violates any law, please contact openaccess@qub.ac.uk. 
Research in Astronomy and Astrophysics manuscript no.

(LTEX: msRAA-2018-0310.R2.tex; printed on February 21, 2019; 1:25)

\title{
Multi-wavelength observations of the 2014 June 11 M3.9 flare: temporal and spatial characteristics
}

\author{
Damian J. Christian ${ }^{1}$, David Kuridze ${ }^{2,3}$, David B. Jess ${ }^{3}$, Menoa Yousefi ${ }^{1}$ and Mihalis \\ Mathioudakis ${ }^{3}$ \\ ${ }^{1}$ Department of Physics and Astronomy, California State University Northridge, Northridge, CA 91330, \\ USA; damian.christian@csun.edu \\ 2 Institute of Mathematics, Physics and Computer Science, Aberystwyth University, Ceredigion, Cymru, \\ SY23 3, UK \\ 3 Astrophysics Research Center, School of Mathematics and Physics, Queenś University Belfast, Belfast \\ BT7 INN, UK
}

Received 2018 month day; accepted 20xx month day

\begin{abstract}
We present multi-wavelength observations of an M-class flare (M3.9) that occurred on 2014 June 11. Our observations were conducted with the Dunn Solar Telescope (DST), adaptive optics, the multi-camera system ROSA (Rapid Oscillations in Solar Atmosphere) and new HARDcam (Hydrogen-Alpha Rapid Dynamics) camera in various wavelengths, such as Ca II K, Mg I b $b_{2}$ (at $5172.7 \AA$ ), and $\mathrm{H} \alpha$ narrow-band, and G-band continuum filters. Images were re-constructed using the Kiepencheuer-Institut Speckle Interferometry Package (KISIP) code, to improve our image resolution. We observed intensity increases of $\approx 120$ $150 \%$ in the $\mathrm{Mg}, \mathrm{Ca} \mathrm{K}$ and $\mathrm{H} \alpha$ narrow band filters during the flare. Intensity increases for the flare observed in the SDO EUV channels were several times larger, and the GOES X-rays increased over a factor of 30 for the harder band. Only a modest delay is found between the onset of flare ribbons of a nearby sympathetic flare and the main flare ribbons observed in these narrow-band filters. The peak flare emission occurs within a few seconds for the $\mathrm{Ca} \mathrm{K}$, $\mathrm{Mg}$, and $\mathrm{H} \alpha$ bands. Time-distance techniques find propagation velocities of $\approx 60 \mathrm{~km} / \mathrm{s}$ for the main flare ribbon and as high as $300 \mathrm{~km} / \mathrm{s}$ for smaller regions we attribute to filament eruptions. This result and delays and velocities observed with SDO $(\approx 100 \mathrm{~km} / \mathrm{s})$ for different coronal heights agree well with the simple model of energy propagation versus height, although a more detailed model for the flaring solar atmosphere is needed. And finally, we detected marginal quasi-periodic pulsations (QPPs) in the 40-60 second range for the Ca K,
\end{abstract}


$\mathrm{Mg}$ and $\mathrm{H} \alpha$ bands, and such measurements are important for disentangling the detailed flarephysics.

Key words: magnetic reconnection-Sun: flares - Sun: atmosphere

\section{INTRODUCTION}

Solar flares vary in magnitude and frequency from rare, large, X-class flares, to common micro-flares and other sub-arcsecond explosive events (Jess et al. 2018). In flares, a rapid energy transfer occurs between the corona, chromosphere, and photosphere through non-thermal electron beams, radiation, conduction, Magnetoacoustic/Alfvén waves, and mass motions. The intermittent nature of the non-thermal electron beams can result in very rapid variations in chromospheric and coronal emission. These variations arise from a combination of energy/ionization imbalance and chromospheric condensation, and are determined by the intensity of the non-thermal energy flux deposited in the lower atmosphere. The chromospheric plasma is heated to very high temperatures (5-30 MK) creating an overpressure, leading to an expansion into the overlying atmosphere. This in turn results in blueshifts in upper chromosphere and transition region lines, a process known as chromospheric evaporation (Milligan et al. 2006; Pevtsov et al. 2007; Milligan et al. 2015). On the other hand, due to the upward and downward momentum balance there should be down flowing pattern (condensation) as a back reaction of chromospheric evaporation, leading a redshifts of tens of kilometers per second in $\mathrm{H} \alpha$ (Kuridze et al. 2015). Additionally, many flares are accompanied by white light emission. White light flare emission is observed in the near-UV and optical continuum, often appearing in transient footpoint regions (Neidig 1989; Isobe et al. 2007; Jess et al. 2008), and see reviews by Hudson et al. (2006); Hudson (2011), for example. The energy content of the white light component of a flare may actually exceed the soft X-ray component by a factor of 100 (Hudson 2011).

There are many open questions on the conditions in the photosphere and chromosphere pre-flare, and their response during the flare. Recent advances in solar imaging in both spatial and temporal resolution allow us to measure the response of the photosphere and chromosphere at multiple heights in the solar atmosphere. Thus, the morphology and temporal changes of the flare emission observed in different narrow-band filters (such as $\mathrm{Ca} \mathrm{K}, \mathrm{Mg}$ ) promise to help constrain the detailed mechanisms of flare energy production and release, e.g. reviews by Fletcher et al. (2011); Hudson (2011). Lawrence et al. (1983), using observations in the $\mathrm{Mg} \mathrm{I} \mathrm{b}_{2}$ line, found $\mathrm{Mg}$ flare kernels resembled white-light flare kernels, and associated the $\mathrm{Mg}$ emission with the impulsive flare phase. More recently, Bai et al. (2014) used $\mathrm{Mg} \mathrm{I}_{2}$ to probe the poorly constrained chromospheric magnetic field. 
In the present paper, we employ these new capabilities of higher spatial and temporal resolution of ground-based instrumentation and report on an M-class flare observed at several layers in the solar atmosphere. We observed an M3.9 flare observed on 2014 June 11 (SOL2014-06-11T21:03 UT), with the Rapid Oscillations of the Solar Atmosphere or (ROSA), HARDcam and CSUNcam instruments at the National Solar Observatory's Dunn Solar Telescope. In Section 2 we present the ROSA and ground-based observations and data analysis, and inclusion of observations from NASA's Solar Dynamic Observatory (SDO). In Section 3 we present results for the flare characteristics, and compare these to previous observations and discuss the flare propagation in simple models and search for quasi-periodic pulsations (QPPs) in Section 4. Lastly, in Section 5 we summarize our findings.

\section{OBSERVATIONS}

Our data were obtained with the Rapid Oscillations in the Solar Atmosphere (ROSA: Jess et al. 2010) (ROSA; Jess et al. 2010) camera system. ROSA is a synchronized, multi-camera high cadence solar imager installed on the Dunn Solar Telescope (DST) at the National Solar Observatory, Sacramento Peak, NM. The DST pointing was at S18.5E60.0, covering AR 12087 (at heliocentric coordinates, $-755^{\prime \prime},-295^{\prime \prime}$ ). The observations sequence started at 19:20 UT, and continued until 21:31 UT, with a span of just over 2 hours and 11 minutes. The ROSA continuum channels recorded $30 \mathrm{fps}$ and narrow-band $\mathrm{Mg}$ I $\mathrm{b}_{2}$ filter was obtained at half of this data rate. The ROSA set-up used with a wider field of view than many previous studies, $\approx 2^{\prime}$ giving a resolution of $0.12^{\prime \prime}$ per pixel or a 2 -pixel diffraction-limited resolution of $\approx 173 \mathrm{~km}$. ROSA was combined with the Hydrogen-Alpha Rapid Dynamics camera (HARDcam) (Jess et al. 2012) and CSUNcam (Grant et al. 2015), using $\mathrm{H} \alpha$ and $\mathrm{Ca}$ II K (hereafter Ca K) filters at a frame rate of 30 fps, respectively. Central wavelengths and filter properties for the $\mathrm{H} \alpha$ and $\mathrm{Ca} \mathrm{K}$ are given in Jess et al. 2010. Interferometric BIdimensional Spectrometer (IBIS; Cavallini 2006; Reardon \& Cavallini 2008) observations using the Na $\mathrm{D}_{1}$ line were also obtain and have been presented by (Kuridze et al. 2016). The observations were obtained with high-order adaptive optics (Rimmele 2004) to correct wavefront deformations in real-time. Although, the overall seeing was poor at times and the observations hampered by clouds, we were able to measure the flare properties and perform speckle image reconstruction. The Kiepenheuer-Institut Speckle Interferometry Package (KISIP) speckle reconstruction algorithm (Wöger et al. 2008), was used on the images, with a 64 to 1 restoration. The $\mathrm{Mg} \mathrm{I} \mathrm{b}_{2}$ (hereafter $\mathrm{Mg}$ ) data was obtained with a cadence of $15.15 \mathrm{fps}$, and the reconstructed cadence is 4.22 seconds per image. The $\mathrm{H} \alpha$ and $\mathrm{Ca} \mathrm{K}$ data were obtained at $30.3 \mathrm{fps}$ and both have a reconstructed cadence of 2.12 seconds per image. We searched for quasi-periodic fluctuations in our narrow-band data using wavelet techniques as described in Jess et al. (2007). Our ground-based observations caught the M3.9 flare rising at $~ 20: 53$ UT and peaking near 20:57 UT, and the GOES X-ray bands measured the flare peak at $\sim 21: 03 \mathrm{UT}$. We show a sequence of flare images in the $\mathrm{Ca} \mathrm{K}, \mathrm{Mg}, \mathrm{H} \alpha$, and G-band in Figure 1. Flares light curves for all 3 bands were extracted in a $34^{\prime} \times 26^{\prime}$ region. 


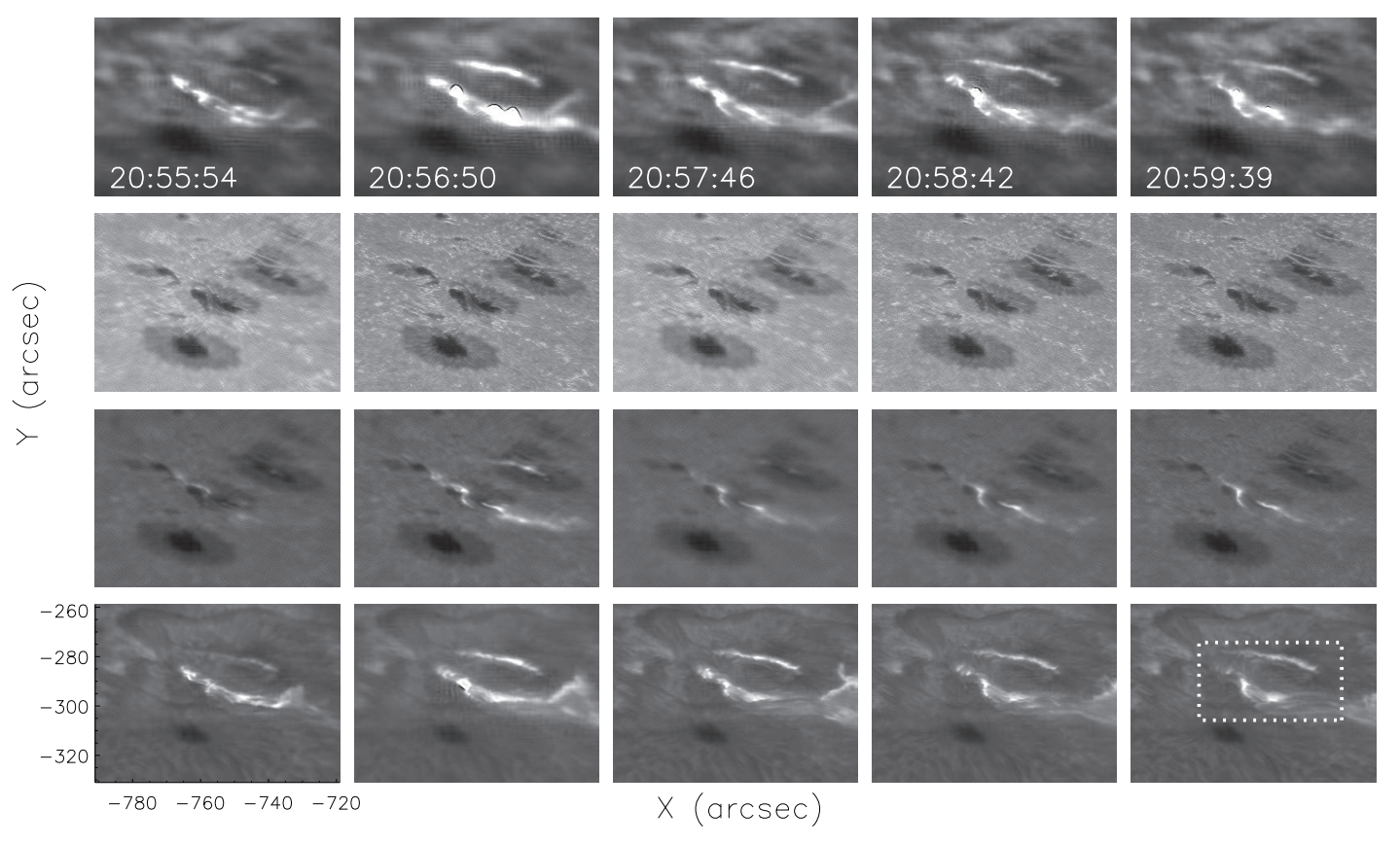

Fig. 1: Image sequence from ground-based observations from ROSA, HaRDcam and CSUNcam spanning the 2014 June 11 M3.9 class flare peak at intervals of about 1 minute. Filters from top to bottom are: Ca K, G-band, $\mathrm{Mg} \mathrm{I} \mathrm{b}_{2}$ and $\mathrm{H} \alpha$. The extraction region for flare light curves is indicated in the lower right panel for $\mathrm{H} \alpha$.

\subsection{Solar Dynamic Observatory Observations}

We further supplemented our ROSA observations with EUV data from the Atmospheric Imaging Assembly (AIA) on-board the Solar Dynamics Observatory (SDO; Lemen et al. 2012) and magnetic information from the Helioseismic and Magnetic Imager (HMI; Scherrer et al. 2012; Schou et al. 2012). The AIA instrument images the entire solar disk in 10 different channels, incorporating a two-pixel spatial resolution of $1.2^{\prime \prime}$ $(\approx 900 \mathrm{~km}$ for the AIA's PSF) and a cadence of $12 \mathrm{sec}$ for the EUV channels and $24 \mathrm{sec}$ for the 1600 and 1700 Åchannels. Here, we selected 5 EUV datasets spanning 20:30 - 22:00 UT on 2014 June 11, consisting of 445 images in each of the 94, 131, 193, 211,304, \& $335 \AA$ AIA channels, and 222 images for the 1600 and $1700 \AA$ channels. The SDO observations caught the M3.9 flare starting at approximately 20:53 UT and the subsequent brightening peaking at near 20:57 for the cooler SDO channel and 21:06 UT for the hotter channels. Light curve for the different SDO channels were extracted in a $120^{\prime \prime} \times 90^{\prime \prime}$ region. Sample SDO images along with our $\mathrm{Mg}$ and $\mathrm{H} \alpha$ bands are shown in Figure 2. We also obtained the HMI line-of-sight (LOS) magnetograms from 20:48 and 21:12 UT, before and after the flare, respectively. The HMI images were aligned and degraded to the AIA resolution with hmi_prep. 

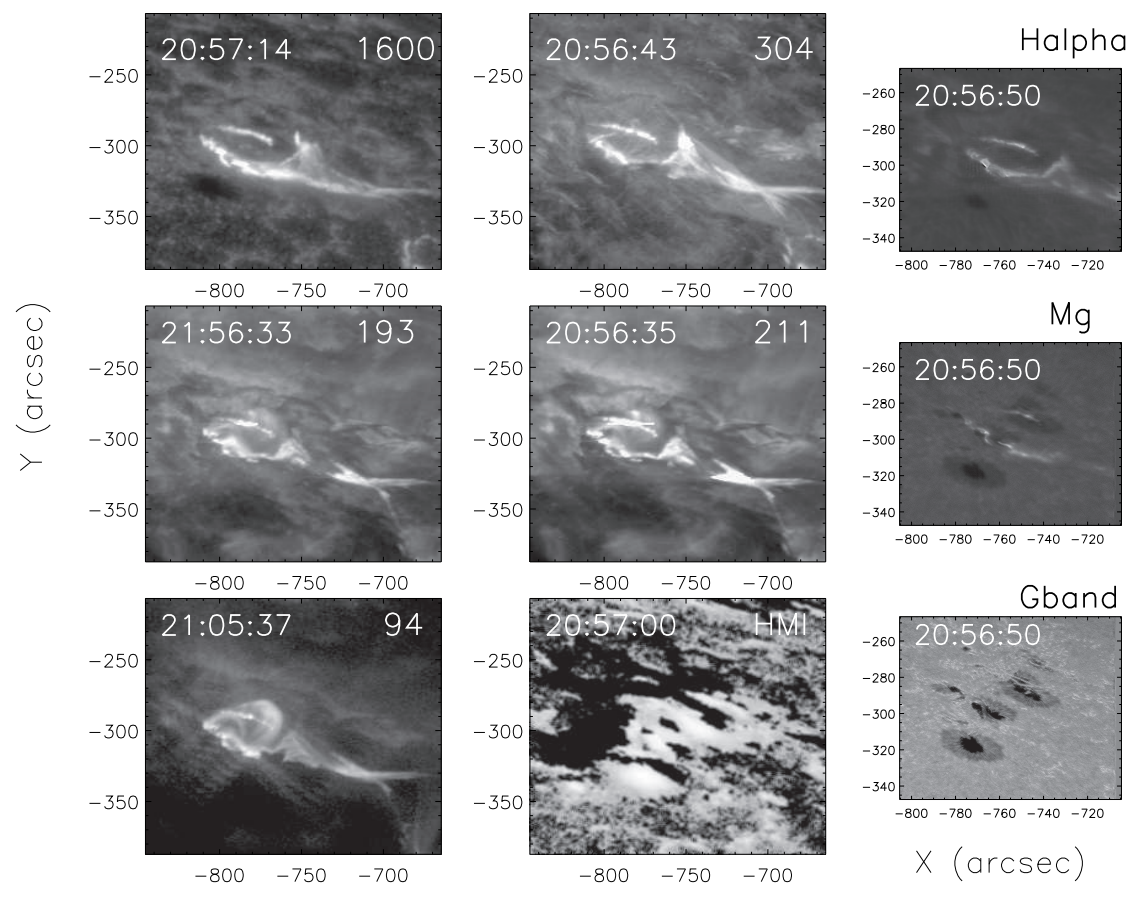

Fig. 2: SDO/AIA flare images in 5 different bands near the flare peak, along with our HARDCam $\mathrm{H} \alpha$, and ROSA G-band and Mg observations in the right column. The HMI LOS magnetogram is also shown in the lower middle panel (see text). (Note: Images were selected closest to the time of the flare peak in each band, but without saturation.)

\section{RESULTS}

\subsection{Flare Properties}

Our multi-wavelength observations of AR 12087 detected an M3.9 flare on 2014 June 11. The GOES soft (1-8 $\AA$ ) and hard $(0.5-4.0 \AA)$ X-rays peaked at 21:03 UT and rose factors of 15 and 33 over the quiescent emission, respectively. The flare in the ROSA Mg I $b_{2}$ light curve rose a factor of 1.24 over the quiescent emission and peaked near 20:56:51 UT. The $\mathrm{Ca} \mathrm{K}$ and $\mathrm{H} \alpha$ light curves increased a factor of 1.55 and 1.5 over their pre-flare, quiescent emission, respectively. In Figure 3 we compare the narrow-band filter data to that obtained from the GOES X-ray satellite. The rise time for the Mg flare was $\sim 105 \mathrm{sec}$, with a decay times of the narrow band filters exceeding 30 minutes and the overall emission stays larger than the preflare quiescent emission for the remaining time of our observations. The GOES peak for our M3.9 flares is $3.9 \times 10^{-5} \mathrm{~W} / \mathrm{m}^{2}$. This works out to a total energy in the 1.0 to $8.0 \AA$ band of $1.1 \times 10^{29} \mathrm{ergs}$ for the 17 minute flare duration. We summarize the flare parameters in different bands in Table 1 extracted from the regions noted in Section 2. 


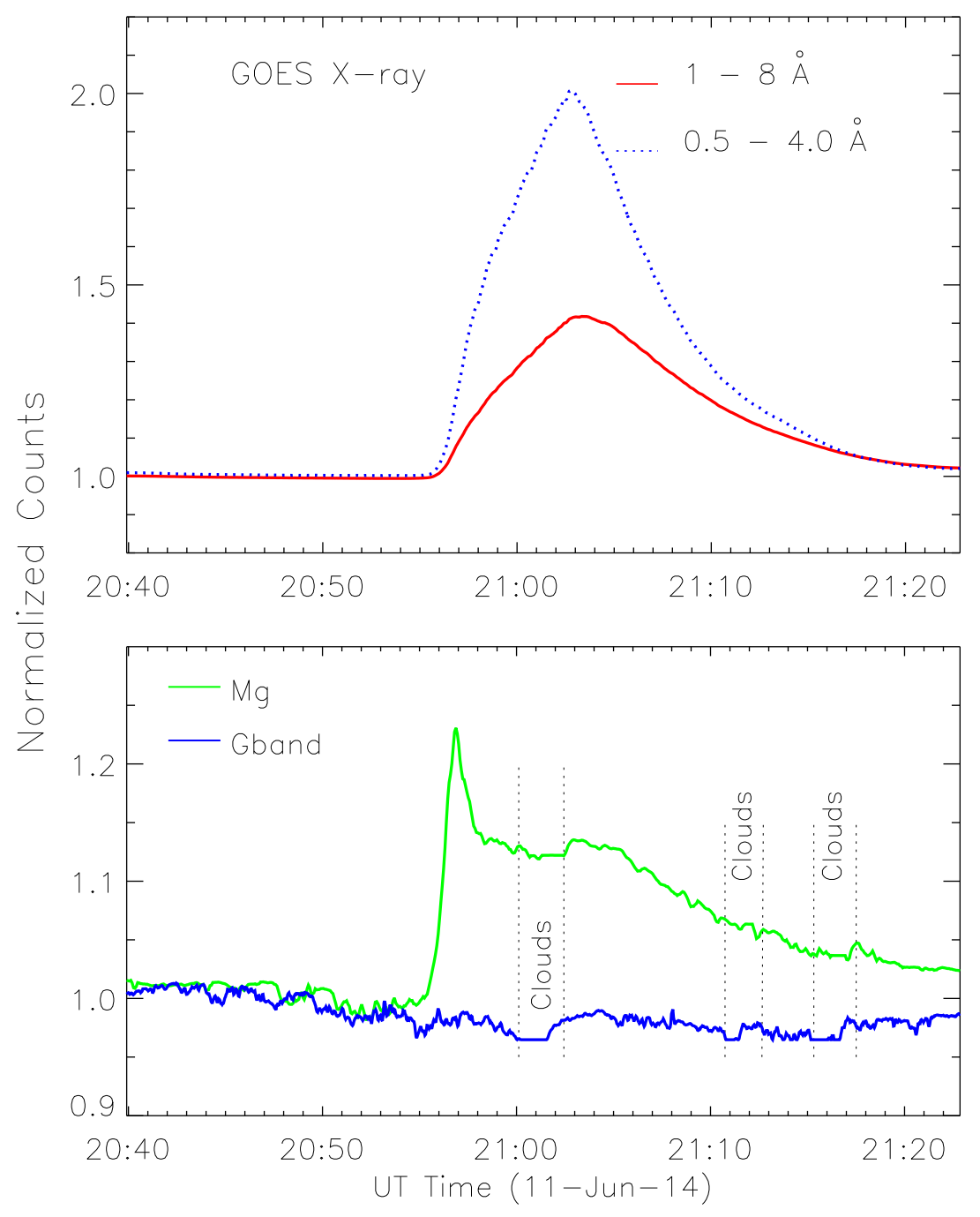

Fig. 3: Sample light curves for the 2014 June 11 M3.9 flare as a function UT times on 2014 June 11 . The top panel shows both the GOES soft X-ray channels with the $0.5-4 \AA$ displayed in blue and the $1.0-8.0 \AA$ in red. The lower panel shows the ROSA Mg (green) and G-band (blue) light curves. Intervals disrupted by clouds are indicated in the lower panel.

\subsection{Flare Morphology}

The flare emerged in the $\mathrm{Ca} \mathrm{K}, \mathrm{Mg}$ and $\mathrm{H} \alpha$ images as 2 separate flare ribbons that evolved into the lower longer flare ribbon ("main" or "lower") with a length of about $25 \mathrm{Mm}$ and a smaller sympathetic flare ribbon ("upper") about $18 \mathrm{Mm}$ long separated by 7 to $10 \mathrm{Mm}$ above. We looked for differences in the flare properties across the flare ribbons in different regions. We show the flare light curves extracted all three bands from these different regions in Figure $4 \mathrm{a}$ along with the flare image for $\mathrm{H} \alpha$. A movie of the $\mathrm{H} \alpha$ images during the flare is also linked to Figure 4a. There was essentially no difference in the rise times 
Table 1: Light Curve Results

\begin{tabular}{|c|c|c|c|c|}
\hline$\lambda$ & $\begin{array}{l}\text { Peak } \\
\text { (UT) }\end{array}$ & $\begin{array}{l}\text { Rise } \\
(\mathrm{min})\end{array}$ & $\begin{array}{r}\text { Decay } \\
(\mathrm{min})\end{array}$ & Peak/Quiescent \\
\hline G-band & No Flare & $\ldots$ & $\ldots$ & $\ldots$ \\
\hline $\mathrm{Ca} \mathrm{K}$ & 20:56:49 & $\sim 1.5$ & $>30$ & 1.55 \\
\hline $\mathrm{Mg}$ & 20:56:51 & $\sim 1.7$ & $\sim 30$ & 1.24 \\
\hline $\mathrm{H} \alpha$ & 20:56:51 & $\sim 1.5$ & $>35$ & 1.50 \\
\hline SDO 304 & $20: 56: 43$ & $\sim 2$ & $\sim 46$ & 4.3 \\
\hline SDO 1600 & 20:56:31 & $\sim 31$ & $\sim 28$ & 4.6 \\
\hline SDO $193^{1}$ & 21:02:42 & $\sim 7$ & $\sim 28$ & 3.4 \\
\hline SDO $211^{1}$ & 20:58:01 & $\sim 2$ & 1.4 & 1.7 \\
\hline SDO $335^{1}$ & 20:56:51 & $\sim 2$ & $\sim 1.5$ & 2.3 \\
\hline SDO $94^{1}$ & 21:04:25 & $\sim 9$ & $\sim 33$ & 12.7 \\
\hline SDO $131^{1}$ & $21: 03: 32$ & $\sim 8$ & $\sim 29$ & 22 \\
\hline Hard X-Ray & 21:03 & $\sim 10$ & $\sim 17$ & 33 \\
\hline Soft X-Ray & 21:03 & $\sim 10$ & $\sim 19$ & 15 \\
\hline
\end{tabular}

${ }^{1}$ Flare peaks occurs several minutes later, consistent with "euv late phase" emission (see text).

between the east and west regions surrounding the lower main flare ribbon. Moving from east to west along the lower flare ribbon find similar rise times, with the largest peak from region labeled box " 5 ". Although in the main flare ribbons, the left region (box labeled "0") rises about 30 seconds before the emission from the upper (sympathetic) flare ribbon (labeled box "7"). The ribbon appears to be erupting along the east-to-west direction, but not in a linear fashion that makes a propagation velocity easily calculated. The more eastward and westward regions showed a more gradual increase than the central regions and only reached a levels $\approx 1.5$ times higher than the quiescent emission, about $40 \%$ lower than the flare peaks for the central regions of the main flare ribbon. In the next section we investigate velocities using time-distance techniques.

\subsection{Velocities}

We attempted to measure the propagation speed of the erupting ribbons in the photosphere and chromosphere during the flare's evolution in the narrow filter bands ( $\mathrm{Ca} \mathrm{K}, \mathrm{Mg}$ and $\mathrm{H} \alpha$ ) using time-distance techniques. Although it is difficult to distinguish a propagation velocity across the flare ribbon, we find a velocity of $\approx 60 \mathrm{~km} / \mathrm{s}$ (shown in Figure $4 \mathrm{~b}$ ) for the expansion at the western end of the main flare ribbon in both $\mathrm{H} \alpha$ and $\mathrm{Ca} \mathrm{K}$. Similar velocities were found for the $\mathrm{Mg}$ band, but were difficult to derive based on its longer cadence, and lower contrast and signal-to-noise. These velocities compare well with previous studies. Huang et al. (2014) found velocities of filament eruptions observed in H $\alpha$ upto $85 \mathrm{~km} / \mathrm{s}$. More puzzling are smaller features moving as fast as $300 \mathrm{~km} / \mathrm{s}$ (blue and orange arrows in $\mathrm{Fg}$ 4b.). These large velocities (over $200 \mathrm{~km} / \mathrm{s}$ ) in an expanding ribbon are unusual, and are most likely associated with erupting filaments. Such large velocities of $340 \mathrm{~km} / \mathrm{s}$ and upto $400 \mathrm{~km} / \mathrm{s}$ were observed in a filament eruption in the SDO 304, 171 and $193 \AA$ bands by Zhu et al. (2015). 

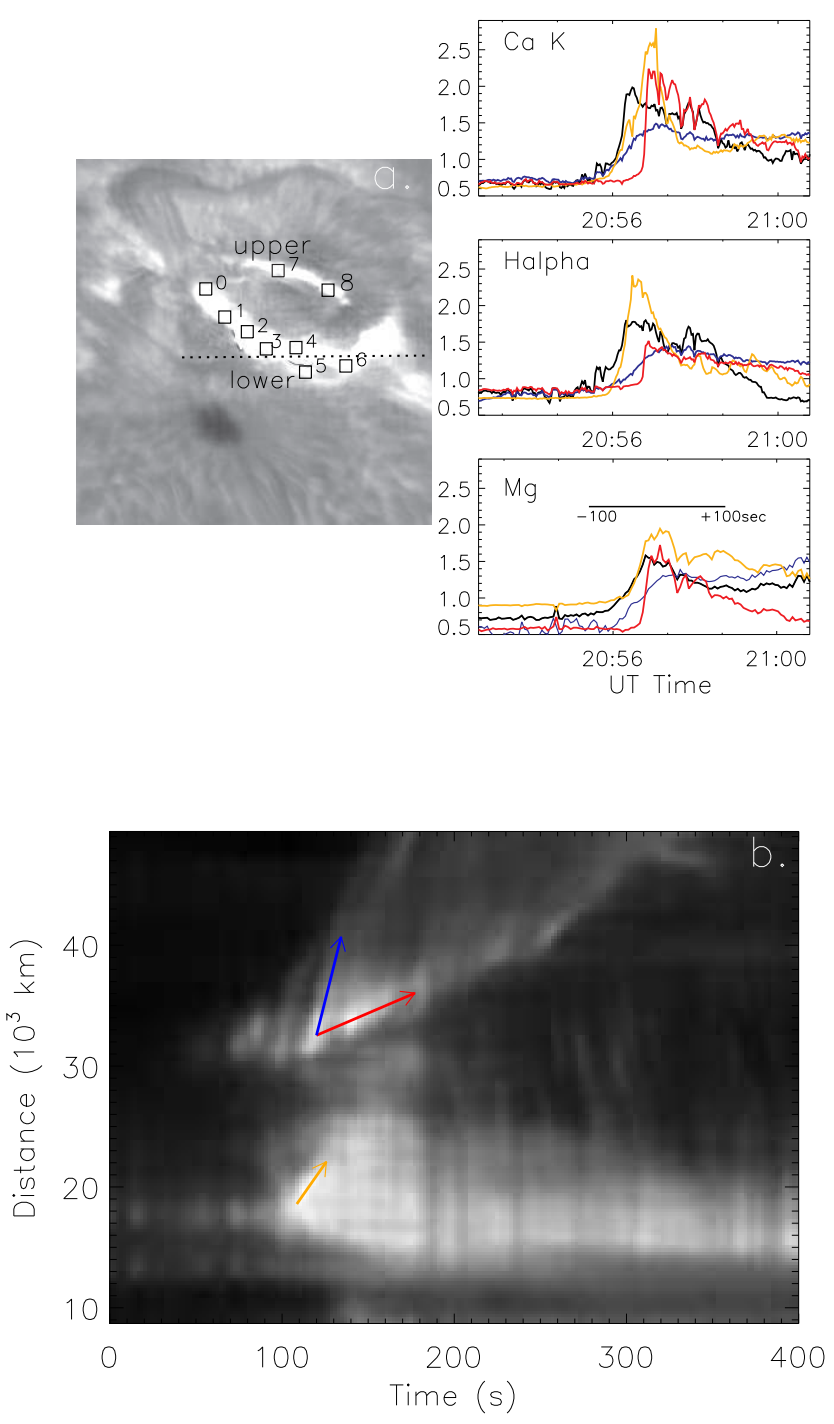

Fig. 4: Shown are comparisons of the $\mathrm{H} \alpha, \mathrm{Ca} \mathrm{K}$ and $\mathrm{Mg}$ light curves extracted from different spatial regions during the M3.9 flare and a time-distance plot for $\mathrm{H} \alpha$. a. (upper panels) The regions the light curves were extracted from are shown in the left upper panel for $\mathrm{H} \alpha$ only. The dashed horizontal line shows where the time-distance plot was taken. The upper left panels show light curves extracted from four selected regions ( 0 - black, 3 - blue, 5 - orange, 7 - red, for ease of presentation), from top to bottom for: $\mathrm{Ca} \mathrm{K}, \mathrm{H} \alpha$, and $\mathrm{Mg}$, respectively. b. (lower panel) Time-distance plot showing motion along the upper and lower flare ribbons for $\mathrm{H} \alpha$. The velocity derived for the western edge of the main ribbon of $\approx 60 \mathrm{~km} / \mathrm{s}$ is indicated with the red arrow, and a higher velocities of $\approx 300 \mathrm{~km} / \mathrm{s}$ for smaller expanding regions is indicated with the blue arrow. The derived velocities in the lower ribbon of $\approx 250 \mathrm{~km} / \mathrm{s}$ is also indicated with the orange arrow. There is a movie of the HARDCam $\mathrm{H} \alpha$ images for Figure 4a (linked in RAA on-line version). The movie starts at 20:55:30 and is shown at a rate of 1 frame per second. The first and last frames are labeled. 


\section{DISCUSSION}

We investigated the properties of an M3.9 flare observing in multiple narrow and continuum band filters and supplemented with EUV imaging data from SDO. Many issues still remain in our understanding of the details of flare physics (densities, temperatures, and magnetic field configurations) that can only be derived from simultaneous observations at high temporal resolution of many layers of the solar atmosphere.

\subsection{Flare Properties}

We observed intensity increases $\approx 120$ to $150 \%$ in the $\mathrm{Mg}, \mathrm{Ca} \mathrm{K}$ and $\mathrm{H} \alpha$ narrow band filters during the flare. Intensity increases for the flare observed in the SDO EUV were several times larger and the GOES $\mathrm{X}$-rays increased a factor of 15 in the soft X-ray band and over a factor of 30 for the harder band. These observed intensity increases in our narrow band filter are slightly lower than those of previous studies (Keys et al. 2011) and may indicate the majority of the flare emission is at hotter temperatures, as observed by the factor of 4-5 times increase observed with SDO.

\subsection{Flare Morphology}

Our observations covered nearly 1 hour before the flare and we are able to observe the detailed morphology of the flare ribbons change on a timescale of seconds. We classify this as a two-ribbon flare showing a lower larger "main" ribbon and an upper smaller region observed in our $\mathrm{Ca} \mathrm{K,} \mathrm{Mg}$, and $\mathrm{H} \alpha$ flare emission. Similar flare morpholgy was observed by Brannon et al. (2015) using IRIS and SDO observaton of an M-class flare. These ribbons of our flare appear to be related and occur within 30 seconds of each other. We find a length of $\approx 25 \mathrm{Mm}$ for the lower main flare ribbon in $\mathrm{H} \alpha$ and $\mathrm{Ca} \mathrm{K}$, and $\mathrm{Mg}$ bands. The flare ribbons are very similar to that of white-light flare emitting regions (Svestka 1976; Dodson-Prince \& Bruzek 1977) and can be compared in our Mg band to the $\mathrm{Mg} \mathrm{I}_{2}$ observations of Lawrence et al. (1983), albeit the 1983 data are at lower spatial resolution.

\subsection{Velocities}

We find almost no difference in the flare's rise times for light curves extracted from regions across the main flare ribbon (Figure 4a) and velocities when comparing various physical regions of the flare using timedistance plots, Also, the rise and decay times between these regions are all very similar (see Figure 4a). The peaks of the $\mathrm{Mg}, \mathrm{Ca} \mathrm{K}$ and $\mathrm{H} \alpha$ emission are all within $\approx 2$ seconds (Figure 5). Typical formation heights for $\mathrm{Ca} \mathrm{K}$ and $\mathrm{H} \alpha$ are $1300 \mathrm{~km}$ and $1500 \mathrm{~km}$, respectively (VAL- Vernazza et al. 1981; FAL90- Fontenla et al. 1990). This would imply a propagation velocity of about $100 \mathrm{~km} / \mathrm{s}$ between these 2 layers, but the uncertainties are large. However, it is curious that the Mg emission, which forms near $700 \mathrm{~km}$ (Schmieder 1979); occurs within seconds of the $\mathrm{Ca} \mathrm{K}$ and $\mathrm{H} \alpha$ emission peaks. This implies during the flare this emission could either be being formed at greater heights, similar to the $\mathrm{Ca} \mathrm{K}$ and $\mathrm{H} \alpha$ formation heights. This would be in agreement for flaring models presented by Mauas et al. (1990). This discrepancy may also result from 


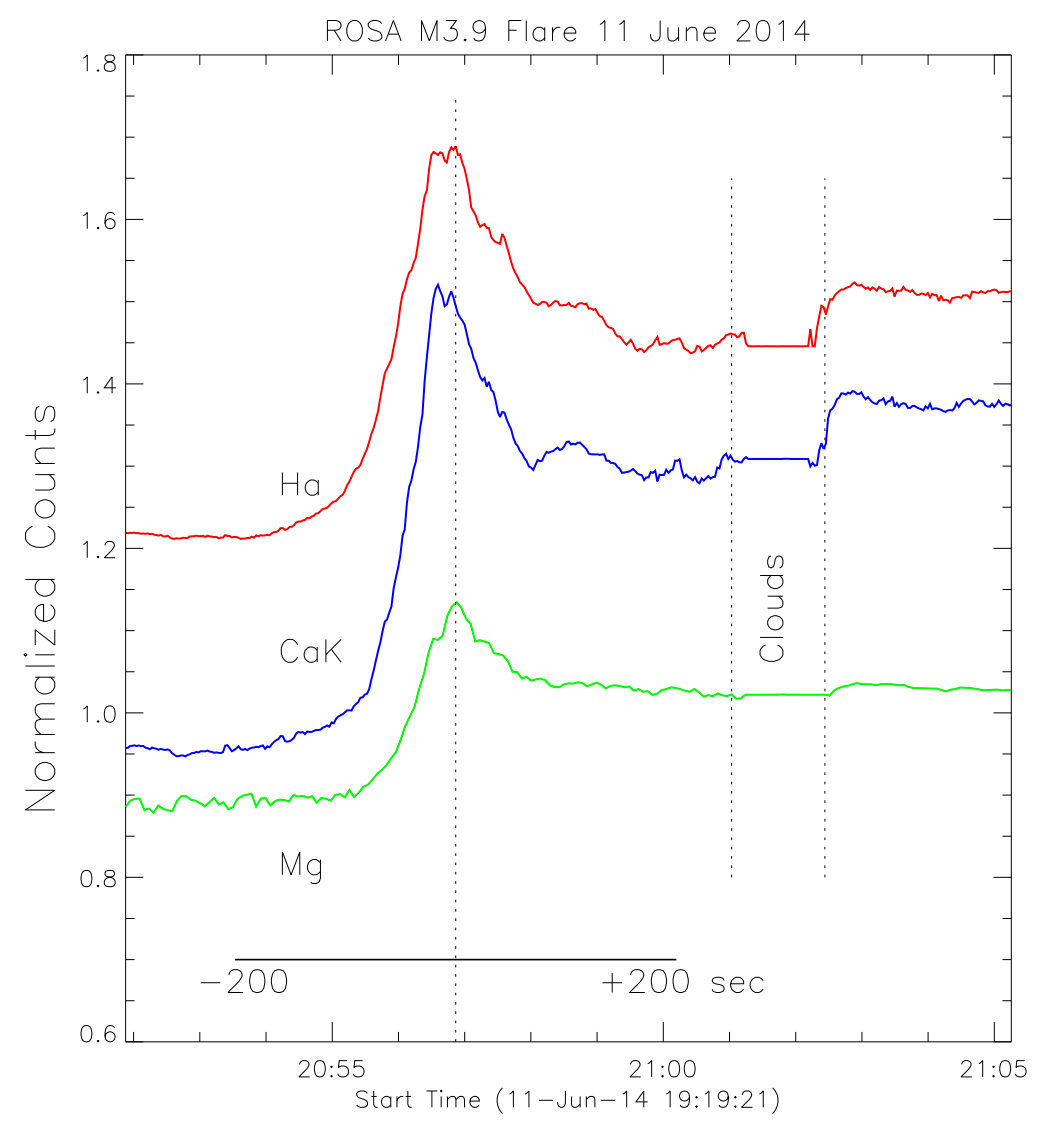

Fig. 5: Light curves for different filters for the M3.9 flare. From top to bottom are the $\mathrm{H} \alpha$ (red), Ca K (blue), and $\mathrm{Mg}$ (green) based plotted in normalized counts ( $\mathrm{Mg}$ is offset for ease of presentation). No significant delay in the flare peak times is observed between the $\mathrm{Mg}, \mathrm{Ca} \mathrm{K}$ and $\mathrm{H} \alpha$ and $\mathrm{Mg}$ peaks and is indicated with a vertical dashed line in addition to intervals affected by clouds (see text).

the width of the Mg filter being sensitive to emission at several depths, and we also realize the formation height of chromospheric spectral lines during the flare are significantly different then when comparing to the VAL model for the quiet Sun.

Similar calculations can be carried out for the SDO data, although we caution that the $12 \mathrm{sec}$ cadence of the AIA EUV channels and the 24 sec cadence of the $1600 \AA$ channel makes the uncertainties in these velocities large, and the SDO filters AIA filters are broadband, capturing emissions from multi-thermal layers with uncertain formation heights. However it gives us a ball-park estimate for propagation velocities 
to compare with the photosphere and chromosphere. We use Reznikova et al. (2012) for SDO's formation heights and find velocities consistent with $\sim 100 \mathrm{~km} / \mathrm{s}$ between emission observed in the SDO 304 \& $1600 \AA$ A channels (formation heights for SDO $304 \& 1600 \AA$ are $2200 \mathrm{~km}$ and $500 \mathrm{~km}$, respectively), and for propagation between the 304 and SDO $171(\Delta \mathrm{H}=1000 \mathrm{~km})$. We can also compare our photospheric and chromospheric measurements to those of the transition region and corona as measured by SDO. We can compare the delay between Ca K and the SDO $304 \AA$ band. These 2 layers have a height difference of $900 \mathrm{~km}$ for the standard VAL model and this results in a velocity of $75 \mathrm{~km} / \mathrm{s}$. If the Ca K formation height is much lower, i.e. $500 \mathrm{~km}$, then the propagation velocity is larger, at $\approx 140 \mathrm{~km} / \mathrm{s}$, a slightly higher velocity than a typical M-class flare, and similar to velocities found for other SDO channels. Again we caution the quiet-Sun VAL model is not applicable for the formation heights of chromospheric spectral lines during the flare. The colder SDO channel (SDO 304 with about 50,000 K) had a longer decay time than a hot SDO channel (SDO 193 with about 1 million K), and as we would have expected the SDO 193 flare peaks much later (up to 6 minutes) than SDO 304, since SDO 193 covers the coronal region and SDO 304 the chromosphere. In Figures 6 we illustrate the light curves for several SDO channels along with our ground-based narrow band channels.

The later emission of the SDO $193\left(\approx 1.5 \times 10^{6} \mathrm{~K}\right)$ and $211 \AA$ channel $\left(\approx 2 \times 10^{6} \mathrm{~K}\right)$, and the delays observed in the hotter channels (94 and $131 \AA$ ) peak upto 8 minutes later than the cooler SDO channels (e.g. 1600, $304 \AA$ ). These later peaks can be attributed the "euv late phase" as described in Woods et al. (2011) and Liu et al. (2013a). This emission may be caused by different higher, but connected loops that have a different thermal history. Figure 6 also includes the light curve for the $335 \AA$ emission that traces Fe XVI at $\approx 2.5 \times 10^{6}$ for comparison to these works. Similar delays for the hotter SDO channels were observed by Cheng et al. (2015) for a two-ribbon M-class flare, a sample of flares presented by Aschwanden \& Shimizu (2013), and the X-class flare observed by Liu et al. (2014). We also note that the GOES bands peaking several minutes later than the narrowband filters and cooler SDO channels is unusual. The GOES 1-8 $\AA$ (SXR) light curve is over-plotted in Figure 6 and more closely aligns with the SDO 335, 94 and $131 \AA$ channels and the "euv late-phase" emission. Such behavior of the GOES SXR flux lagging emission from the lower solar atmosphere has been observed before for between it and: the TRACE $1600 \AA$ emission for an M8.0 flare observed by Liu et al. (2013b), SDO $1600 \AA ̊$ emission for a C3.2 flare observed by Qiu et al. (2013), and with the $\mathrm{H} \alpha$ emission for a 44.1 flare observed by Deng et al. (2013). The early impulsive phase of our M3.9 flare observed in narrow band filters ( $\mathrm{CaK}, \mathrm{H} \alpha, \mathrm{Mg}$ ), and cooler SDO bands (304 and $1600 \AA$ ) is readily attributed to rapid heating by non-thermal electron beam precipitation, however the lagging SXR flux is harder to explain. Liu et al. (2013b) using an OD EBTEL model, invoke continuously expanding ribbons, emitting in the UV create new flare loops by continuous magnetic reconnection and continue to heat the corona. Qiu et al. (2013) call upon the cooling of hot plasma that has been evaporated into the corona to explain this later phase. The GOES SXR is sensitive to temperatures over 5MK $(\log \mathrm{T}=6.7)$ and its later peak agrees well with those observed in the SDO 94, 131 and $335 \AA$ bands, which are sensitive to emission at temperatures greater than $2.5 \mathrm{MK}(\log \mathrm{T} \approx 6.4)$. Future $\mathrm{X}$-ray solar flare observations need 


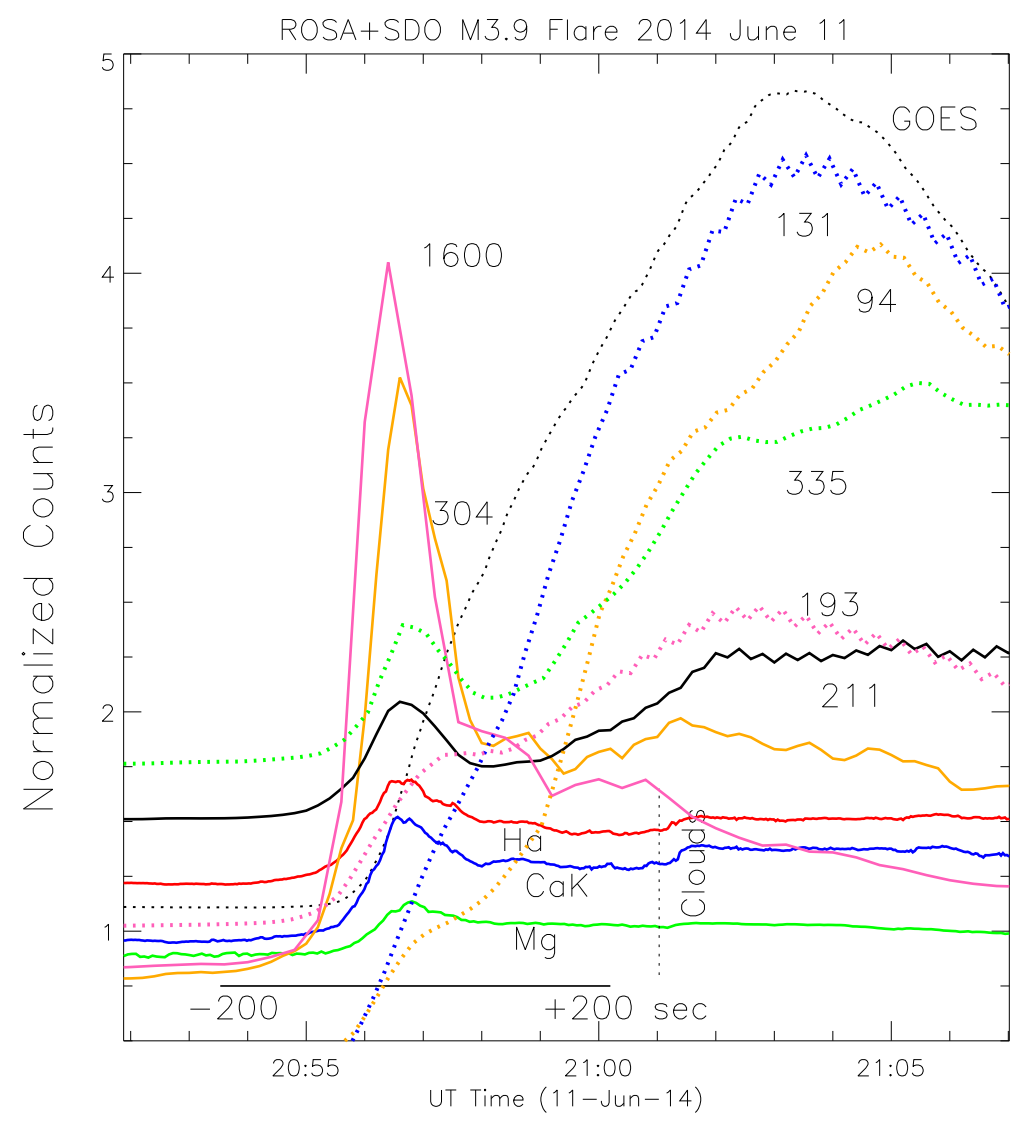

Fig. 6: Shown are the light curves for the cooler $(193,211,304, \& 1600 \AA)$, hotter $(94,131, \& 335 \AA$ ) SDO channels, the GOES soft X-ray band (1-8 ̊̊; SXR), and our CaK (blue), Mg (green) and H $\alpha$ (red) narrowband filter data. The SDO AIA channels shown are: 304 (orange), 1600 (pink), 211 (black), 193 (pink, dotted line), 335 (green, dotted line), 94 (orange, dotted line), and 131 Å (blue, dotted line). Individual light curves are normalized by their average counts and are offset for ease of presentation. The re-scaled GOES soft X-ray band (SXR; 1.0 - $8.0 \AA$ A) is over-plotted (black, dotted line) and aligns with the "euv late-phase" emission (see text).

to include high spatial and temporal resolution of the lower solar atmosphere to better model such flare behavior. 


\subsection{Search for Quasi-periodic pulsations}

We searched for quasi-periodic pulsations (QPP) in our narrow-band filter data using wavelet and Fourier techniques described in Jess et al. (2007). We found a suggested QPP with periods in the 40-60 second range for the $\mathrm{CaK}, \mathrm{Mg}$ and $\mathrm{H} \alpha$ bands. We present the results of this analysis in Figure 7 for $\mathrm{H} \alpha$ (selected from the largest region used in the other analysis for maximum single to noise, although simialr periods are derived for the smaller regions shown in Figure 4a), including the de-trended light curve, wavelet and Fourier power, and confidence level. No detections of white light QPP have been found in solar flares and QPP in narrow-band filters have only been suggested. QPPs of about 60 seconds were found after a C-class flare by Keys et al. (2011), and more recently, Kolotkov et al. (2015) found quasi-harmonic behavior of QPP observed in an X3.2 flare with intrinsic modes in the 15 to 100 seconds range, and Brannon et al. (2015) found periods of $\approx 140$ seconds in an M-class flare. The mechanism for QPP are still currently under debate, but involves some form of oscillatory reconnection in the magnetic loops or in MHD modes (McLaughlin et al. 2012). More recently, quasi-period slipping was observed during an X-class flare using IRIS and SDO data (Li \& Zhang 2015). The associated periods were found in the 3 to 6 minutes and attributed to tearing mode instability generating oscillatory reconnection that may ultimately be driven by solar p-modes.

\section{SUMMARY AND CONCLUSIONS}

We have presented multi-wavelength observations of the 2014 June 11 M3.9 flare. We observed intensity increases $\approx 120-150 \%$ in the $\mathrm{Mg}, \mathrm{Ca} \mathrm{K}$ and $\mathrm{H} \alpha$ narrow band filters during the flare. Intensity increases for the flare observed in the SDO EUV were several times larger ( $\approx 4$ times for $304 \AA$ with $\mathrm{T} \approx 50,000 \mathrm{~K})$, and the GOES X-rays increased over a factors of 15 and 33 for the soft and hard bands, respectively. The flare morphology observed in these narrow-band filters shows a main flare ribbon and a sympathetic flare ribbon about 7 to $10 \mathrm{Mm}$ away. Only modest delays are found between the onset of emission across the main flare ribbon and upto 30 seconds between the main flare regions and of the nearby sympathetic flare (upper region). The peak flare emission occurs within a few seconds for the $\mathrm{Ca} \mathrm{K}, \mathrm{Mg}$, and $\mathrm{H} \alpha$ bands. Timedistance techniques find propagation velocities of $\approx 60 \mathrm{~km} / \mathrm{s}$ for the main flare ribbon and velocities as high as $300 \mathrm{~km} / \mathrm{s}$ in smaller regions of the main flare ribbon that we attribute to filament eruptions. Propagation velocities between the chromosphere and coronal layers, although uncertain, are found to be as large as $\approx 100 \mathrm{~km} / \mathrm{s}$. These results and delays and velocities for different coronal heights observed with SDO are consistent with this velocity, and agree well with the simple model of energy propagation versus height, although a more detailed model for the flaring solar atmosphere is needed. Hotter SDO channels show emission with delays of upto 8 minutes and our consistent with the "euv late phase" observed for several Mand X- class flares. And finally, we detected marginal quasi-periodic pulsations (QPPs) in the 40-60 second range for the $\mathrm{Ca} \mathrm{K}, \mathrm{Mg}$ and $\mathrm{H} \alpha$ bands, and such measurements are currently lacking in the literature, but are important for disentangling the detailed flare-physics. Future high time resolution observations of solar flares (such as those available with DKIST) are important for disentangling the detailed flare-physics. 


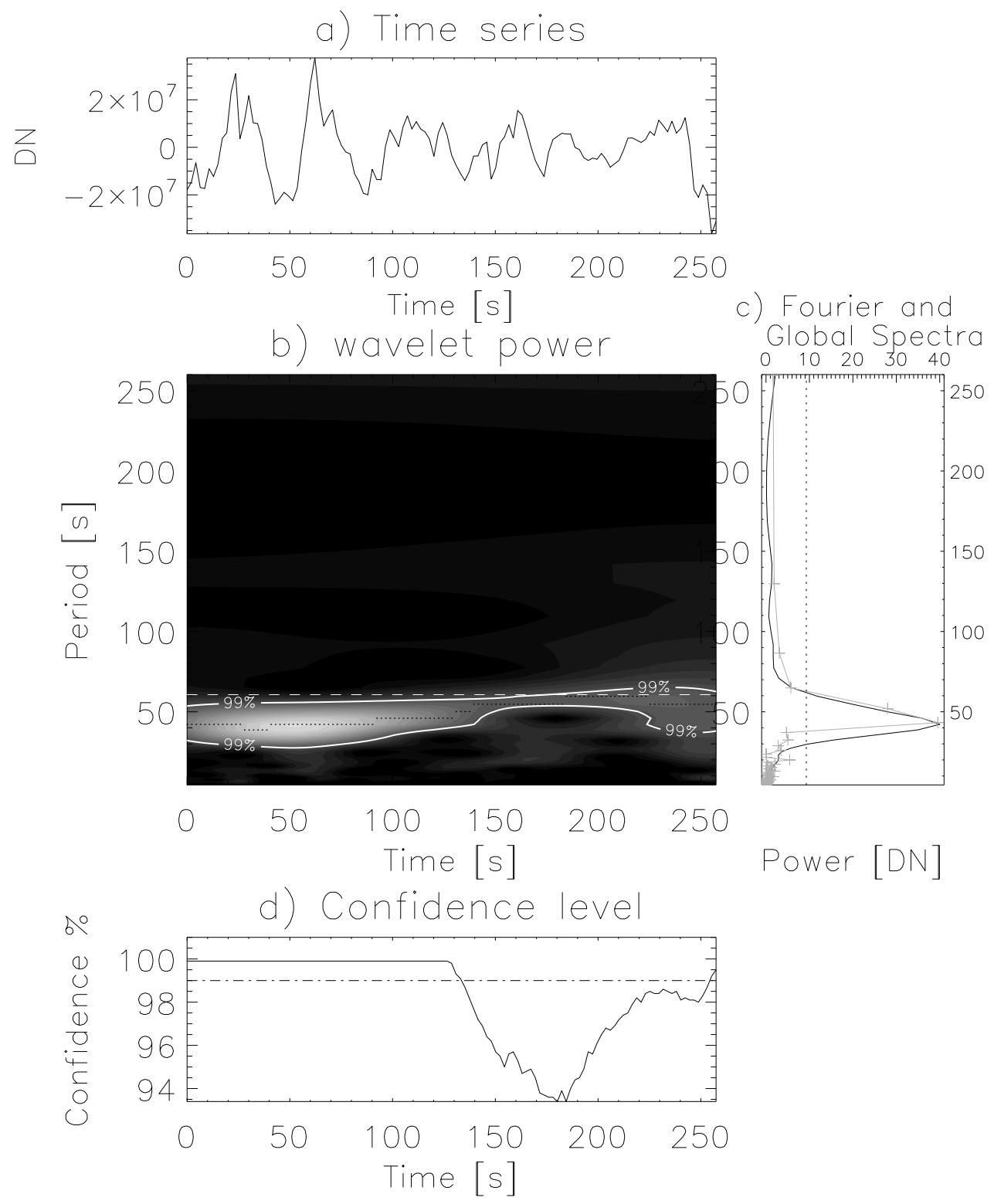

Fig. 7: Temporal analysis of the de-trended $\mathrm{H} \alpha$ filter light curve. a. The top diagram shows the original $\mathrm{H} \alpha$ light curve for $\approx 4$ minutes after the flare peak. b. Shown is the wavelet power transform along with locations where detected power is at, or above, the $99 \%$ confidence level are contained within the contours. c) The right-hand-side plot shows the summation of the wavelet power transform over time (full line) and the Fast Fourier power spectrum (crosses) over time, plotted as a function of period. Both methods show marginal detections in the 40-60 sec range. The global wavelet (dotted line) and Fourier (dashed dotted line) $95 \%$ significance levels are also plotted. The lowest panel, d. shows the probability levels $(1-p) \times$ 100 as discussed in Sect. 4.4.

Acknowledgements We thank the NSO Dunn Solar Telescope staff, including Doug Gilliam for their excellent support for this project, and we dedicate this paper to the late Michael Bradford who's keen eye pointed out this solar flare. The work of D.K. was supported by Sêr Cymru II Part-funded by the 
European Regional Development Fund through the Welsh Government. D.K. acknowledge support from Georgian Shota Rustaveli National Science Foundation project FR17_323. We thank an anonymous referee for suggested improvements to the manuscript.

\section{References}

Aschwanden, M., Shimizu, T. 2013, ApJ, 776, 132

Bai, X.-Y., Deng, Y.-Y., Su, J.-T., Wang, G.-P. 2014, Res. in Astronomy \& Astrophysics, 14, 193

Cavallini, F., 2006, Solar Physics, 236, 415

Brannon, S. R., Longcope, D. W., Qiu, J. 2015, ApJ, 810, 4

Cheng, X., Hai, Q., Ding, M. D., Liu, K., Chen, P. F., Fabg, C., Liu, Y.D. 2015, ApJ, 809, 46

Deng, N. et al. 2013, ApJ, 769, 112

Dodson-Prince, H. W., \& Bruzek, A. 1977, in Illustrated Glossary for Solar and Solar-Terrestrial Physics, ed. A. Bruzek \& C. J. Durrant (Dordrecht: Reidel), 81

Fletcher, L., Dennis, B. R., Hudson, H. S. et al. 2011, SSRv, 59, 19

Fontenla, J. M., Avrett, E., \& Loeser, R. 1990, ApJ, 355, 700 ( FAL90)

Grant, S. D., Jess, D. B., Moreels, M. G. et al. 2015, ApJ, 806, 132

Huang, Z., Madjarska, M. S., Koleva, K., Doyle, J. G., Duchlev, P., Dechev, M., Reardon, K. 2014, A\&A, 566,148

Hudson, H. S., Wolfson, C. J., Metcalf, T. R. 2006, Sol. Phy. 234, 79

Hudson, H. 2011, Space Sci. Rev., 158, 5

Isobe, H., Kubo, M., Minoshima, T. et al. 2007, PASJ, 595, 807

Jess, D. B., Mathioudakis, Erdélyi, R., Verth, G., McAteer, R. T. J., Keenan, F. P. 2007, ApJ, 680, 1523

Jess, D. B., Mathioudakis, ErM., Crockett, P. J., Keenan, F. P. 2008, ApJ, 688, 119

Jess, D. B., Mathioudakis, M., Christian, D. J., Keenan, F. P., Ryans, R. S. I., Crockett, P. J. 2010, Solar Phys., 261, 363

Jess, D. B., De Moortel, I., Mathioudakis, M., Christian, D. J., Reardon, K. P., Keys, P. H., Keenan, F. P. 2012, ApJ, 757, 160

Jess, D. B., Dillon, C. J., Kirk, M. S. et al. 2018, ApJ in press and arXiv:1812.06978

Keys, H. P., Jess, B. D., Mathioudakis, M., Keenan, P. F. 2011, A\&A, 529, 127

Kolotkov, D. Y., Nakariakov, V. M., Kupriyanova, E. G., Ratcliffe, H., Shibasaki, K. 2015, A\&A, 574, 53 ; QPP

Kuridze, D., Mathioudakis, M.,Simões, P. J. A. et al. 2015, ApJ, 813, 125

Kuridze, D., Mathioudakis, M., Christian et al. 2016, ApJ, 832, 147

Lawrence, K. J., Chapman, A. G., Herzog, D. A. 1983, Solar Physics, 89, 341

Lemen, J. R., Title, A. M., Akin, D. J., et al. 2012, Sol. Phys., 275, 17

Li, T., Zhang, J. 2015, ApJL, 804, L8

Liu, K., Zhang, J., Wang, Y., Cheng, X. 2013, ApJ, 768, 150 
Liu, W.-J., Qui, J., Longcope, D. W., Caspi, A. 2013, ApJ, 770, 111

Liu, R., Titov, V. S., Gou, T., Wang, Y., Liu, K., Wang, H. 2014, ApJ, 790, 8

Mauas, P. J. D., Machado, M.E., Avrett, E. H. 1990, ApJ, 360, 715

McLaughlin, J. A., Thurgood, J. O., MacTaggart, D. 2012, A\&A, 548, 98

Milligan, R. O. et al. 2006, ApJL, 638, 117

Milligan, R. O. 2015, Sol. Phys., 290, 3399

Neidig, D. F. 1989, Solar Phys., 121, 261

Pevtsov, A.A., Balasubramaniam, K. S., Hock, Rachel A. 2007, Advances in Space Research, 39, 1781

Qiu, J., Sturrock, Z., Longcope, D. W., Klimchuk, J. A., Liu, W.-J. 2013, ApJ, 774, 14

Reardon, K.P., Cavallini, F. 2008 A\&A, 481, 897

Reznikova, V. E., Shibasaki, K., Sych, R. A., Nakariakov, V. M. 2012, ApJ, 746, 119

Rimmele, T. R., 2004, Proc. SPIE, 5490, 34

Scherrer, P. H., Schou, J., Bush, R. I., Kosovichev, A. G. et al. 2012, Sol. Phys., 275, 207

Schmieder, B. 1979, Astronomy and Astrophysics, 74, 273

Schou, J., Scherrer, P. H., Bush, R. I., et al. 2012, Sol. Phys., 275, 229

Svestka, Z. 1976, Solar Flares (Berlin: Springer)

Vernazza, J. E., Avrett, E. H., \& Loeser, R. 1981, ApJS, 45, 635 (VAL)

Wöger, F., von der Lühe, O., Reardon, K. 2008, A\&A, 488, 375

Woods, T. N., Hock, R., Eparvier, F. et al. 2011, ApJ, 739, 59

Zhu, C., Liu, R., Alexander, D., Sun, X., McAteer, R. T. 2015, ApJ, 813, 60 